\title{
Cor triventriculare sinistrum illustrated by cardiac MRI
}

\author{
Florian Sagmeister ${ }^{1}$, Karin Mild ${ }^{1}$, Thorsten Lieberknecht ${ }^{2}$ and Meinrad Beer ${ }^{1 *}$ \\ ${ }^{1}$ University Hospital Ulm, Department of Diagnostic and Interventional Radiology, Ulm, Germany \\ ${ }^{2}$ Herzklinik Ulm, Dr. Haerer und Partner, Ulm, Germany
}

\section{Introduction}

Cor triventriculare is a rare congenital anomaly of the heart which was first described in the year 1954 by Hakkila et al. [1]. The partial subdivision of one ventricle in two chamber was described for both the right and left ventricle. The separation in the left ventricle is called cor triventriculare sinistrum (CTS). Aim of our case report is to illustrate this rare anomaly by different cardiac MRI-sequences.

\section{Case report}

We report about a 25-year female patient who suffered from a singular syncope after sports activity. ECG showed a supraventricular tachycardia with extra-systoles, a CTS was diagnosed by transesophageal echocardiography. When in follow up a cardiac MRI was performed, clinical symptoms and cardiac arrhythmias had subsided.

The patient was examined with a 3-Tesla scanner (Skyra, Siemens, Germany). For anatomic information, axial T2 haste black blood sequences of the whole thorax and T1 TSE dark blood sequences in short axis direction were acquired, for functional information, short axis und long axis SSFP -CINE sequences. Then, intracardiac flow pattern were detected by time resolved 3D-TWIST-angiography (MRA). Finally, tissue integrity was analyzed by inversion recovery late gadolinium enhancement (LGE) in long and short axis direction.

The abnormal trabecularization of the left ventricle, ranging from the midventricular parts to the apex were depicted by all sequences. Focal thinning of the pericardium near the apex as possible sign of focal dysplasia or aplasia was at best demonstrated by T2 Haste and CINE. There was no cardiac hypertrophy with a normal end-diastolic septal wall thickness of $8 \mathrm{~mm}$. CINE quantified a slight dilatation of the left ventricle in the end-diastolic and end-systolic frame (LVEDD $60 \mathrm{~mm}$, LVESD $44 \mathrm{~mm}$, LVEDVI $112 \mathrm{ml} / \mathrm{m}^{2}$ ) and a slight reduction of global left (LVEF 47\%) and right ventricular function (RVEF 44\%). Concerning regional function, basal and midventricular cardiac segments were hypokinetic, one focal apicolateral segment almost akinetic. MR-angiography demonstrated an almost symmetrical inflow into the two apical parts of the LV with a slightly earlier filling of the lateral cavity. LGE revealed no pathological contrast enhancement in the hypokinetic / akinetic regions and excluded a thrombus formation in the hypertrabecularized left ventricular apex (Figures 1-5).

\section{Discussion}

There are several reports of a cor triventriculare of the right ventricle [1-3], but only few previous reports concerning a CTS. Cohen et al. reported about a subdivision of left ventricle with a cardiac thrombus in their autopsy-study [4]. There is only one available case report of a "cor triventriculare sinistrum" in a cardiac MRI-examination [5].

In the present case report, the demonstration of abnormal trabecularization with a separation of a small apical "third ventricle" was possible by using different MRI-sequences. Our findings of an apicolateral hypokinesia to akinesia, the focal aplasia of the pericardium, the slight enlargement of left ventricle and the slight reduction of left ventricular function are all in agreement with the former MRI-report by Merkle et al. [5]. The missing pathological LGE in the hypokinetic regions underlines the thesis, that the reduced
A

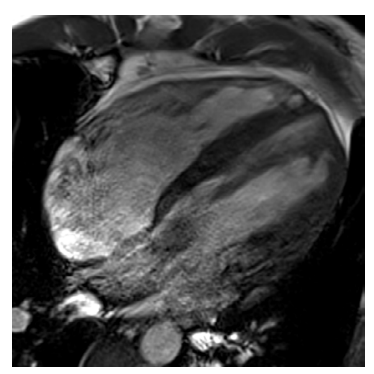

$\mathrm{C}$

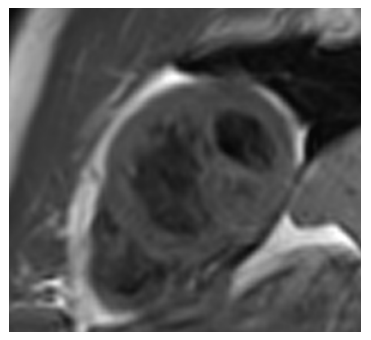

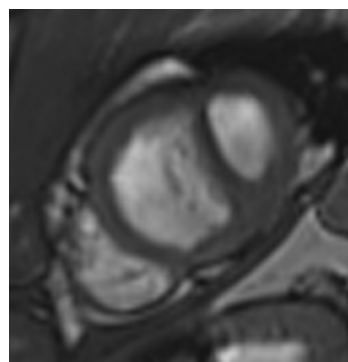

$\mathrm{D}$

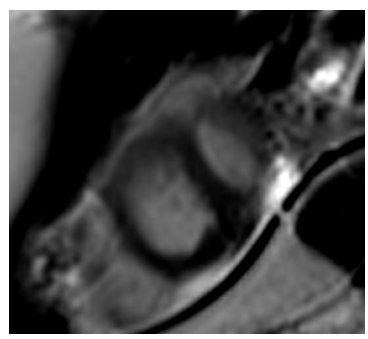

Figure 1. The depiction of the pathological apical third ventricle was possible using long(1A, 4-chamber-view) and short axis (1B) CINE-sequences. Furthermore, the abnormal trabecularization could be identified in short axis T1 TSE dark blood (1C) and short axis LGE-sequences (1D).

Correspondence to: Meinrad Beer, University Hospital Ulm, Department of Diagnostic and Interventional Radiology, Albert-Einstein-Allee 23, D-89081 Ulm, Germany, Tel: +49 / 731 / 500-61001; Fax: +49 / 731 / 500-61002; E-mail: meinrad.beer@uniklinik-ulm.de

Special Issue: Imaging of myocardial diseases: to new horizons

Valentin E. Sinitsyn

Professor

Department of Radiology

Federal Center of Medicine and Rehabilitation

Russia

E-mail: vsini@mail.ru

Published: October 18, 2016 
A
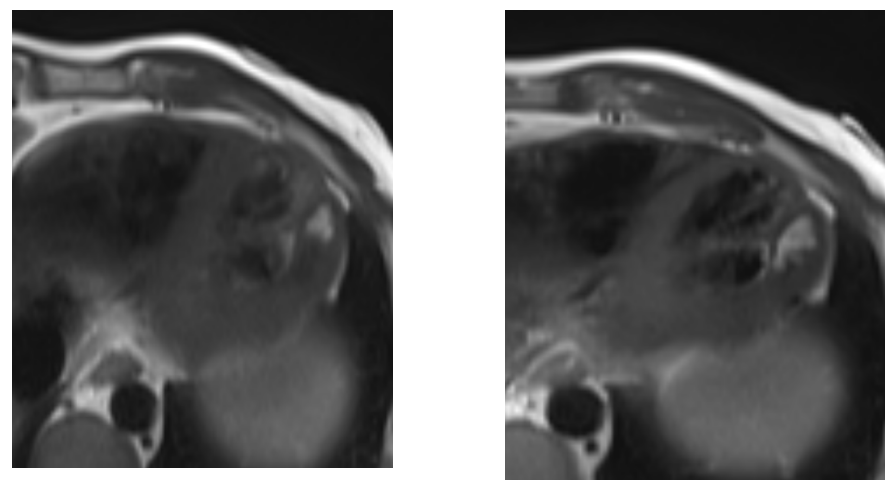

Figure 2. Increased signal in the apicolateral "third ventricle" in T2 Haste dark blood as a possible sign of reduced or turbulent flow in this area.
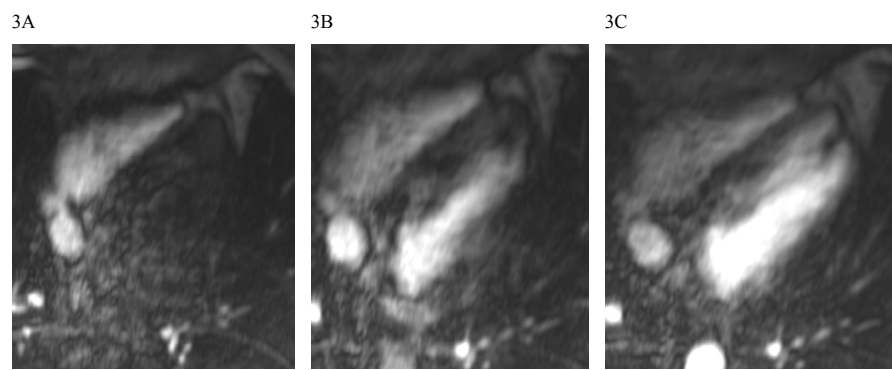

Figure 3. Dynamical time resolved TWIST in the original dataset (Figure 3 ) and in two variants of MIP-reconstructions (Figures 4 and 5).

$4 \mathrm{~A}$

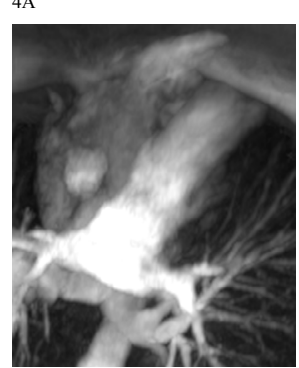

$4 \mathrm{~B}$

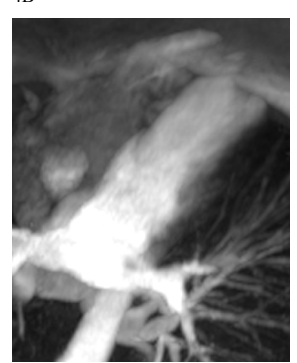

$4 \mathrm{C}$

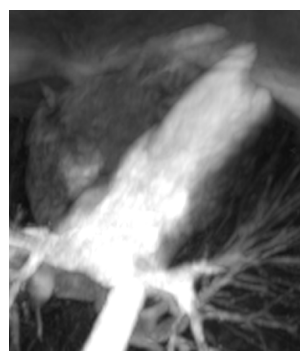

Figure 4. Dynamical time resolved TWIST in the original dataset (Figure3) and in two variants of MIP-reconstructions (Figures 4 and 5).

$5 \mathrm{~A}$

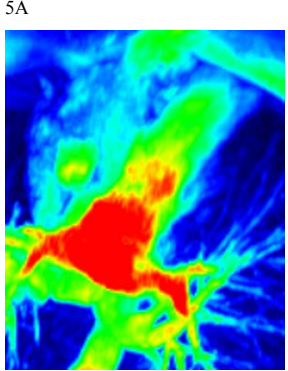

$5 \mathrm{~B}$
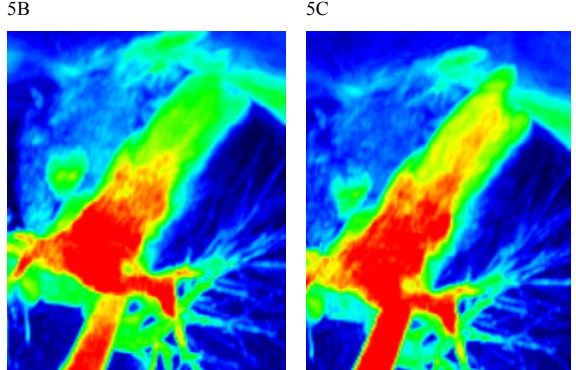

Figure 5. Dynamical time resolved TWIST in the original dataset (Figure3) and in two variants of MIP-reconstructions (Figures 4 and 5). wall motion in the affected area is not a result of previous ischemia or infarction. Moreover, cardiac ischemia would be very uncommon in this young patient age.

In contrast to Merkle et al. we did not find mitral valve insufficiency and in contrast to the report of Cohen et al. our apical separating structure has not the typical signal intensity of a thrombus and is more likely a hypertrophied trabecle [4].

Dark-blood T2-Haste sequences revealed increased signal intensity in the apicolateral region of the "third ventricle" which could be a sign of reduced or turbulent flow in this area. TWIST-angiography helped to visualize the almost symmetrical inflow into both apical cavities. LGE-imaging excluded thrombus formation in both apical chambers.

\section{Conclusion}

This case report shows the value of cardiac MRI for a comprehensive evaluation of cor triventriculare sinistrum. Adding the new tools of time resolved MR-angiography and LGE imaging for intracardiac flow and tissue integrity analysis, cardiac MRI turns out to be the ideal tool in description and follow-up for patients with CTS.

\section{References}

1. Hakkila R, Hakkila J (1954) Cor triventriculare, complete dextroposition of the aorta, infundibular pulmonary stenosis, ventricular septal defect and patent foramen ovale in an adult. Ann Med Intern Fenn 43: 171-6.[Crossref]

2. Watler D, Wynter L (1961) Cor triventriculare: infundibular stenosis with subdivision of the right ventricle. Br Heart $J$ 23: 599-602.[Crossref]

3. Konwaler B (1944) Cor triventricular. AHJ 27: 259-65.

4. Cohen L, Roberts W (1961) Acquired cor triventriculare, a rare complication of cardiomyopathy. AHJ 73: 538-41.[Crossref]

5. Merkle N, Burgstahler C, Kunze M, Loeffler C, Hombach V (2006) Cor triventriculare with pericardial aplasia and mitral valve prolapse: a novel congenital dysplastic syndrome? Eur Heart J 27: 1288. [Crossref]

Copyright: $@ 2016$ Sagmeister F. This is an open-access article distributed under the terms of the Creative Commons Attribution License, which permits unrestricted use, distribution, and reproduction in any medium, provided the original author and source are credited. 